\title{
NOTE ON NONNEGATIVE MATRICES
}

\section{D. ž. DJOKOVIĆ ${ }^{1}$}

Abstract. Let $A$ be a nonnegative square matrix and $B=$ $D_{1} A D_{2}$ where $D_{1}$ and $D_{2}$ are diagonal matrices with positive diagonal entries. Several proofs are known for the following theorem: If $A$ is fully indecomposable then $D_{1}$ and $D_{2}$ can be chosen so that $B$ is doubly stochastic. Moreover, $D_{1}$ and $D_{2}$ are unique up to a scalar factor. It is shown that these results can be easily obtained by considering a minimum of a certain rational function of several variables.

Several recent papers [1], [2], [3], [4] were devoted to the following problem: Given a nonnegative square matrix $A$, find the conditions for the existence of two diagonal matrices $D_{1}$ and $D_{2}$ such that $D_{1} A D_{2}$ is doubly stochastic. We shall show that it is related to a simple minimum problem. This leads to a short proof of Theorem (6.1) of [1] which avoids the use of Menon's operator.

We begin with some definitions. An $n \times n(n \geqq 2)$ matrix $A$ is reducible if there exists a permutation matrix $P$ such that

$$
P A P^{T}=\left(\begin{array}{ll}
A_{1} & 0 \\
B & A_{2}
\end{array}\right)
$$

where $A_{1}$ is a $k \times k$ matrix, $1 \leqq k \leqq n-1$. Otherwise we say that $A$ is irreducible.

An $n \times n(n \geqq 2)$ matrix $A$ is fully indecomposable if there do not exist permutation matrices $P$ and $Q$ such that

$$
P A Q=\left(\begin{array}{ll}
A_{1} & 0 \\
B & A_{2}
\end{array}\right)
$$

where $A_{1}$ is a $k \times k$ matrix, $1 \leqq k \leqq n-1$.

TheOREM. Let $A$ be a nonnegative $n \times n$ fully indecomposable matrix. Then there exist diagonal matrices $D_{1}$ and $D_{2}$ with positive diagonals such that $D_{1} A D_{2}$ is doubly stochastic. Moreover $D_{1}$ and $D_{2}$ are uniquely determined up to scalar multiples.

For the proof we need the following

Received by the editors August 23, 1969.

AMS Subject Classifications. Primary 1560, 1565.

Key Words and Phrases. Nonnegative matrix, doubly stochastic matrix, irreducible matrix, fully indecomposable matrix.

1 Supported in part by NRC Grant A-5285. 
Lemma. Let $A$ be a nonnegative $n \times n$ matrix. Then $A$ is fulty indecomposable if and only if there exist permutation matrices $P$ and $Q$ such that $P A Q$ has a positive main diagonal and is irreducible.

A short proof of this lemma appears in [1].

Proof of THE THEOREM. By the lemma we can assume that $A=\left(a_{i j}\right)$ has positive main diagonal and is irreducible. Let

$$
f\left(x_{1}, \cdots, x_{n}\right)=\prod_{k=1}^{n}\left(\sum_{i=1}^{n} a_{k i} x_{i}\right) / \prod_{k=1}^{n} x_{k}
$$

the variables being restricted by

$$
x_{k}>0 \quad(1 \leqq k \leqq n), \quad \sum_{k=1}^{n} x_{k}=1 .
$$

Let $\left(b_{i}\right)$ be a boundary point of the region (1) and, for instance, $b_{1}=\cdots=b_{s}=0, b_{k}>0(s<k \leqq n)$. Since $A$ is irreducible we infer that at least one entry $a_{i j}>0$ for $1 \leqq i \leqq s, s<j \leqq n$. This implies that $f\left(x_{1}, \cdots, x_{n}\right) \rightarrow+\infty$ when $\left(x_{k}\right) \rightarrow\left(b_{k}\right)$. Therefore $f$ attains its minimum in some point $\left(c_{k}\right)$ of the region (1). The partial derivatives of $f$ vanish at $\left(c_{k}\right)$ since $f$ is homogeneous. Hence,

$$
\sum_{k=1}^{n} c_{j} a_{k j}\left(\sum_{i=1}^{n} a_{k i} c_{i}\right)^{-1}=1 \quad(j=1, \cdots, n)
$$

which proves the first assertion of the theorem.

For the uniqueness it is sufficient to prove the following assertion: If the matrices $X=\left(x_{i j}\right), D_{1}=\operatorname{diag}\left(d_{1}^{\prime}, \cdots, d_{n}^{\prime}\right), D_{2}=\operatorname{diag}\left(d_{1}^{\prime \prime}, \cdots, d_{n}^{\prime \prime}\right)$ satisfy

(i) $X$ is irreducible doubly stochastic with positive elements on the main diagonal;

(ii) $d_{i}^{\prime}>0, d_{i}^{\prime \prime}>0(1 \leqq i \leqq n)$;

(iii) $D_{1} X D_{2}$ is doubly stochastic, then $D_{1}$ and $D_{2}$ are scalar matrices. Since

$$
\begin{aligned}
& \sum_{j=1}^{n} d_{i}^{\prime} x_{i j} d_{j}^{\prime \prime}=1 \Rightarrow\left(\max d_{i}^{\prime}\right)\left(\min d_{j}^{\prime \prime}\right) \leqq 1, \\
& \sum_{i=1}^{n} d_{i}^{\prime} x_{i j} d_{j}^{\prime \prime}=1 \Rightarrow\left(\max d_{i}^{\prime}\right)\left(\min d_{3}^{\prime \prime}\right) \geqq 1 .
\end{aligned}
$$

we conclude that none of these inequalities is strict. This implies that $x_{r_{s}}=0$ whenever $d_{r}^{\prime}=\max d_{i}^{\prime}$ and $d_{s}^{\prime \prime}>\min d_{j}^{\prime \prime}$ or $d_{r}^{\prime}<\max d_{i}^{\prime}$ and $d_{s}^{\prime \prime}=\min d_{j}^{\prime \prime}$. This contradicts (i) unless $D_{1}$ and $D_{2}$ are scalar matrices.

The proof is completed. 


\section{REFERENCES}

1. R. A. Brualdi, S. V. Parter and H. Schneider, The diagonal equivalence of a nonnegative matrix to a stochastic matrix, J. Math. Anal. Appl. 16 (1966), 31-50. MR 34 \#5844.

2. M. V. Menon, Reduction of a matrix with positive elements to a doubly stochastic matrix, Proc. Amer. Math. Soc. 18 (1967), 244-247. MR 35 \#6708.

3. R. Sinkhorn, A relationship between arbitrary positive matrices and doubly stochastic matrices, Ann. Math. Statist. 35 (1964), 876-879. MR 28 \#5072.

4. R. Sinkhorn and P. Knopp, Concerning nonnegative matrices and doubly stochastic matrices, Pacific J. Math. 21 (1967), 343-348. MR 35 \#1617.

University of Waterloo, Ontario, Canada 